\title{
Workload-Driven Placement of Column-Store Data Structures on DRAM and NVM
}

\author{
Robert Lasch \\ robert.lasch@tu-ilmenau.de \\ TU Ilmenau, SAP SE \\ Walldorf, Germany \\ Thomas Legler \\ thomas.legler@sap.com \\ SAP SE \\ Walldorf, Germany
}

\author{
Robert Schulze \\ robert.schulze@sap.com \\ SAP SE \\ Walldorf, Germany \\ Kai-Uwe Sattler \\ kus@tu-ilmenau.de \\ TU Ilmenau \\ Ilmenau, Germany
}

\begin{abstract}
Non-volatile memory (NVM) offers lower costs per capacity and higher total capacities than DRAM. However, NVM cannot simply be used as a drop-in replacement for DRAM in database management systems due to its different performance characteristics. We thus investigate the placement of column-store data structures in a hybrid hierarchy of DRAM and NVM, with the goal of placing as much data as possible in NVM without compromising performance. After analyzing how different memory access patterns affect query runtimes when columns are placed in NVM, we propose a heuristic that leverages lightweight access counters to suggest which structures should be placed in DRAM and which in NVM. Our evaluation using TPC-H shows that more than $80 \%$ of the data touched by queries can be placed in NVM with almost no slowdown, while naively placing all data in NVM would increase runtime by $53 \%$.
\end{abstract}

ACM Reference Format:

Robert Lasch, Robert Schulze, Thomas Legler, and Kai-Uwe Sattler. 2021. Workload-Driven Placement of Column-Store Data Structures on DRAM and NVM. In International Workshop on Data Management on New Hardware (DAMON'21), June 20-25, 2021, Virtual Event, China. ACM, New York, NY, USA, 8 pages. https://doi.org/10.1145/3465998.3466008

\section{INTRODUCTION}

Non-volatile memory (NVM) represents an emerging technology which offers exciting opportunities for the design and optimization of data structures and data placement strategies. NVM fills the gap in the memory hierarchy between DRAM and SSDs. Compared to SSDs, it offers low latency and byte-addressability, while also providing high capacities and persistence in comparison to volatile DRAM. While possible use cases of NVM's persistence feature for data management have been extensively studied [1, 3, 4, 9, 14, 16, 17], the usage of NVM in this paper is motivated purely by its lower cost per capacity and the larger possible total capacities relative to DRAM. For example, at the time of writing, a system with 3

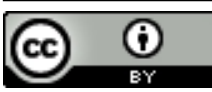

This work is licensed under a Creative Commons Attribution International 4.0 License.

DAMON'21, fune 20-25, 2021, Virtual Event, China

(c) 2021 Copyright held by the owner/author(s).

ACM ISBN 978-1-4503-8556-5/21/06.

https://doi.org/10.1145/3465998.3466008

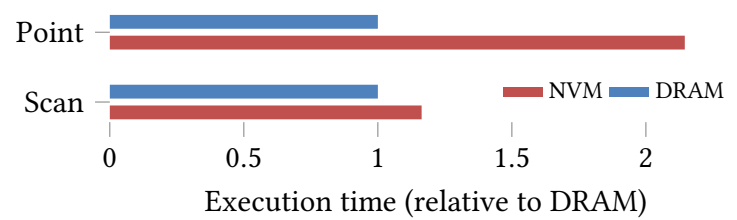

Figure 1: Scan and point queries are affected differently by placing tables in NVM instead of DRAM.

TB of DRAM and 6 TB of Intel Optane NVM costs the same as a system with only 6 TB of DRAM [23]. This is a $50 \%$ higher memory capacity at the same cost. NVM thereby presents an opportunity to reduce costs while simultaneously enabling larger workloads if it can be used instead of DRAM.

In this paper we thus investigate using NVM for storing SAP HANA's read-optimized column-store data structures. The representation of columns in this column store as two distinct data structures, data vectors, and dictionaries, presents an opportunity for placing data at the granularity of these data structures and exploiting their access characteristics. Also, placing read-optimized data on NVM is a good fit, as NVM can be read faster than it can be written [12]. Our goal is to move as much data to NVM as possible without negatively affecting query performance in analytical workloads. For this, the different access characteristics of NVM compared to DRAM need to be considered: Currently available NVM technology has roughly $3 \mathrm{x}$ higher random access latency, and about $3 \mathrm{x}$ lower bandwidth [12]. In the preliminary experiment shown in Figure 1, we found that these characteristics affect point accesses much more than they affect sequential scans: For the experiment, we ran two different queries on TPC-H's LINEITEM table using the SAP HANA instance described in Section 4.1: A Point query that groups on L_ORDERKEY and computes the sum of L_EXTENDEDPRICE, and a Scan query that counts the number of rows matching a greaterthan predicate on the L_EXTENDEDPRICE column. Both queries are repeated multiple times. As the names indicate, the first query performs many point accesses on the dictionaries of the two referenced columns to materialize values for grouping and summing, while the second query only performs a single binary search in the dictionary of L_EXTENDEDPRICE, followed by a full scan of the data vector to count the matching rows (see Section 2). The figure shows that both queries execute slower when the data they read is placed in NVM. 
However, the runtime of the scan-heavy query increases by only $16 \%$ whereas the point query is slowed down by $115 \%$ when the data is placed in NVM. This can likely be attributed to lower latencies for the sequential loads [12] primarily done by the scan-heavy query and to effective prefetching. Because the performance between point accesses and scans on DRAM and NVM is so different, we believe that it is crucial to take the dominant access type on a data structure into account when deciding whether it should be placed in NVM. To that end, we propose the use of access counters that distinguish between point and scan accesses at the data structure level to guide placement decisions.

\section{BACKGROUND AND RELATED WORK}

In the following we present the relevant data structures of SAP HANA followed by a brief discussion of related work.

SAP HANA Column Store. SAP HANA stores each table using a read-optimized main and a write-optimized delta fragment [11]. Both are merged periodically, typically when the delta exceeds a certain size. The main store, which we focus on here as it represents the majority of the table data, employs domain encoding for data compression and to speed up accesses. A domain-encoded column consists of a data vector and dictionary. The dictionary contains the sorted and unique original column values, and it supports lookups of values based on their identifiers (called value ids), as well as searches for specific values, that return the respective value id, if present. The data vector stores a value id for each row of the column. Data vectors are bit-packed by default but may also be compressed using logical compression techniques like run-lengthencoding or sparse coding. This can be accompanied by reshuffling the table rows so that the overall table compression becomes more efficient [13]. String dictionaries may also be compressed to further reduce memory usage [15]. Scans on data vectors are accelerated using SIMD instructions [22]. During query execution, there are two main ways how the data vectors and dictionaries are accessed: To obtain a filtered set of rows (SQL WHERE), the dictionary is first searched for matching values and a set of value ids is generated. The sorted nature of dictionaries can be exploited in many cases (e.g., equality- or range-predicates) to avoid full scans of the dictionary. The row ids are then obtained by scanning the data vector for value ids that match the ones produced by the previous dictionary search. The second main access case is when row values need to be materialized, e.g., for projections, joins, or aggregations. In this case, a lookup in the data vector is performed using a row id, which produces a value id that is then looked up in the dictionary to retrieve the value.

NVM Support in SAP HANA. Initial NVM support was added to SAP HANA in 2017 [2]. This feature allows placing all main fragments into NVM, but it does not consider differentiating between data vectors and dictionaries. In contrast, we will show that better cost-performance trade-offs can be achieved by using more fine-granular placement strategies.

NVM-Centric Data Management. Several works focus on designing or optimizing data structures specifically for use with NVM $[3,9,17]$ or redesigning DBMS components and architecture with NVM's persistence features in mind $[4,14,16]$. In this paper, we focus on efficiently placing existing, read-optimized storage data structures of an industry-strength DBMS in NVM, motivated solely by NVM's lower cost and superior capacity relative to DRAM.

NVM in the Storage Layer. For integrating NVM in the storage layer, several works chose buffer-managed approaches [5, 10, 20], where NVM is used as an additional tier in the cache hierarchy. These buffer-managed approaches base their eviction and migration policies on when a page was last accessed, and may duplicate data across DRAM and NVM due to the nature of their policies. We consider storing data in NVM as a placement problem where data resides either in NVM or in DRAM and propose to differentiate between scan and point accesses on data structures to guide the placement decision. Although we implement placement for buffermanaged data structures to simplify the implementation, our results could be transferred to SAP HANA's in-memory structures.

Data Management on Multi-Tier Hierarchies. Besides NVM, several works also focus on placing data across other levels of the memory hierarchy. Early work by Bhattacharjee et al. [6] explores how a multi-tier storage hierarchy with HDDs and SSDs can be used effectively for data management. They statically place database objects on the SSD instead of the HDD by estimating access time savings based on counting random and sequential accesses to disk-pages for a given workload. Boissier et al. [7] make placement decisions between DRAM and secondary storage at columngranularity based on whether the access patterns on columns are predominantly sequential or random. A different storage layout is then used for data placed in secondary storage. We also distinguish between random point and sequential scan accesses in this paper, albeit for placing in-memory data in DRAM or NVM, without changing the storage layout. Vogel et al. [21] present Mosaic, a storage engine that can place data optimally in a tier-less pool of storage devices for scan-heavy workloads in a disk-based system. We aim to provide similarly optimal placement recommendations for in-memory storage.

\section{DERIVING A PLACEMENT POLICY}

The focus of this paper is to investigate placement alternatives between NVM and DRAM for read-optimized column store data structures, with the goal of finding a placement policy that places as much data on NVM as possible without compromising query performance. We discuss our approach to finding such a policy in this section, by first discussing how we enable placement of data in NVM, followed by an initial experiment to assess the feasibility of placing data in NVM. Then, we identify dominant access types using access counters on the two main data structures in SAP HANA, data vectors and dictionaries. Finally, we derive a heuristic for placement decisions on the data structures of individual columns based on the access counters.

\subsection{Facilitating Placement on NVM}

We prototypically implemented data structure placement by extending SAP HANA's buffer cache [19] with a NVM-backed layer. With this extension, a placement hint can be specified that the requested page shall be located in NVM instead of DRAM when a page is requested from the buffer cache. Whether the page is in 


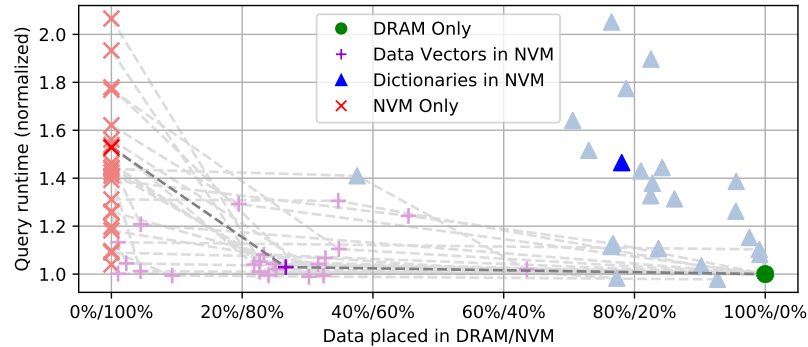

Figure 2: Initial results for TPC-H SF 100 using simple placement strategies based on the type of data structure. The desaturated entries show the runtimes of each of TPC-H's 22 queries, the fully saturated entries show the runtime for a full run of all 22 queries. Dashed lines represent the Pareto frontier for each query and the full run.

DRAM or NVM is then completely transparent to the data structure and algorithms using that page. This results in great flexibility for accommodating individual data structures of columns in either DRAM or NVM. This approach also simplified our initial implementation, as all NVM allocations are performed in a single place in the buffer cache, rather than in each data structure which would need to be modified individually. Although we place at the granularity of data structures in this paper, our approach is equally capable of placing data with page granularity. We use the NVM block provider described in [2] to make allocations in NVM. The block provider uses a Direct Access (DAX)-enabled filesystem to expose blocks allocated on NVM DIMMs configured in App Direct mode as regular memory pointers. By not committing the allocated blocks, the NVM memory can be used as working memory and is properly freed when the system is restarted. Because we are interested in analyzing only the effects of placing data structures in NVM, rather than analyzing NVM as an additional layer in the cache hierarchy (as studied in $[5,20]$ ), we size the buffer cache in our experiments such that it is able to hold the entire working set without evictions. For the same reason, we run all experiments with the accessed data already preloaded into the buffer cache. We discuss further details on the hardware and software configuration for all following experiments and our evaluation in Section 4.1.

\subsection{Placement Based on Data Structure Type}

To gain an initial understanding of the feasibility and potential of placing SAP HANA's main storage in NVM, we tested four simple placement strategies using queries from the TPC-H benchmark with scale factor 100 . The four initial strategies are: DRAM Only $(O)$, i.e., all data is placed in DRAM, Data Vectors in NVM (+), Dictionaries in NVM ( $\mathbf{A})$, and NVM Only $(\times)$. Note that, besides data vectors and dictionaries, the NVM Only strategy also places indices in NVM. This is equivalent to SAP HANA's existing NVM feature [2], except for the fact that our approach operates on page-loadable data.

Figure 2 illustrates for each of the four simple placement strategies query runtimes relative to placing all data in DRAM, as well as the amount of data placed in NVM or DRAM as a fraction of the total amount of data touched by the respective query. This is shown for each of TPC-H's 22 individual queries and for a combined run of all queries. Note that, because only data that is actually read by queries is loaded into the buffer cache, columns that are not touched by the queries (e.g., L_COMMENT) are not included in the memory usage statistics. In other words, all data that is placed in NVM is accessed at least once by the queries. Looking at the results, it is clear that placing all data in NVM $(x)$ is not an acceptable strategy - we observe a 53\% slowdown for the combined workload compared to DRAM-only placement (O), with worst-case slowdowns of up to $106 \%$ for individual queries. We also observe that placing only data vectors in NVM $(+)$ produces acceptable results: a significant fraction of the data is placed in NVM yet query times increase only moderately for most queries. In contrast, placing dictionaries in NVM $(\boldsymbol{\Delta})$ generally results in drastic slowdowns and lower amounts of data stored in NVM. Even among these four basic placement strategies, placing dictionaries in NVM is almost never Pareto-optimal with respect to time and space usage as the dashed lines in Figure 2 show. This is expected given how data is filtered and materialized in HANA (cf. Section 2). For data vectors, scans of large parts are the norm, while dictionaries are primarily subject to random point access. Scan-like access patterns suffer less performance degradation when data is located in NVM than random access, as demonstrated in Figure 1. Nevertheless, for some queries, placing dictionaries in NVM is the better choice. For example, for Q11, placing data vectors in NVM yields a 30\% slowdown, while placing dictionaries in NVM slows the query down by only $3 \%$.

Overall, the initial results demonstrate significant potential of data placement at data structure granularity. In many cases more than $70 \%$ of the data volume can be accommodated in NVM with query slowdowns of less than $20 \%$. However, we also observe that no single strategy works universally well for all workload queries. While placing only data vectors in NVM already yields very good results, additionally placing selected dictionaries in NVM may allow for even lower DRAM usage at similar performance. In the following, we thus explore more fine-grained data placement with a strategy that also takes workload characteristics into account, in particular access patterns on individual columns.

\subsection{Analyzing Access Types}

To understand the workload better and to guide a more refined placement strategy, we now implement access counters on the two main data structures considered in this paper, data vectors and dictionaries. These counters represent the total number of accesses to entries of the data structures, and therefore they closely approximate the actual memory accesses performed by lookup and scan operations. Note that, because data vectors and dictionaries may additionally be compressed, the counters do not accurately reflect actual memory accesses in all cases. We nevertheless implement the access counters at the level of logical accesses since this reduces implementation effort and runtime overhead over a lower-level implementation, while we found logical counting still sufficiently accurate for our purposes. To be able to place at column and datastructure granularity, we collect access counters separately for each column. As we have previously identified a significant performance difference in NVM between point accesses and scans, we distinguish between these two access types for the counters. For point accesses, 


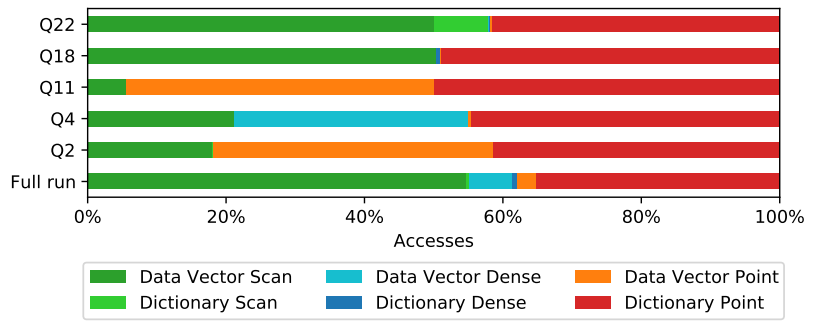

Figure 3: Access types for selected TPC-H queries.

we additionally track dense point accesses. A point access is considered dense if it reads the element prior or subsequent to the data structure's last accessed entry. This distinction is useful because dense point accesses constitute a much more scan-like access pattern than true random point access, which should result in a less significant slowdown when data with this access pattern is placed in NVM. Unlike more advanced approaches which maintain statistics at block level, e.g., with the goal of automated physical database design [8], we only count the total number of accesses to each data structure as we are currently only interested in making placement decisions at data structure granularity. We point out that only one set of counters (three integers) exists per data vector and per dictionary. Counters are also updated lazily, i.e., if multiple accesses occur in succession, these are first aggregated locally by the plan operator and added to the counter of the respective data structure only once. The performance and space overhead of maintaining counters is therefore negligible.

Figure 3 shows the distribution of access types on all columns collected for a run of all TPC-H queries and selected interesting queries. For the majority of the 22 queries, we observe that data vectors are predominantly scanned or accessed with dense point accesses whereas dictionaries are mostly accessed using point accesses, and among these point accesses, dense point accesses are the exception. Dictionaries are also almost never scanned. One counterexample is Q22 where the dictionary of C_PHONE is scanned to perform substring matching. In Figure 3, we also show the access types for queries Q2 and Q11 which exhibit many data vector point accesses. For both queries, around $40 \%$ of the total accesses are data vector point accesses, which coincides with the results of Section 3.2 where we observed a more significant slowdown compared to other queries when data vectors of Q2 and Q11 were placed in NVM.

It is also interesting that the majority of point accesses on data vectors are dense point accesses, i.e., exhibit a scan-like access pattern. An extreme example of this is Q4, where $55 \%$ of all accesses are data vector point accesses but $61 \%$ of these accesses are dense. This is caused by fully materializing the L_RECEIPTDATE and L_COMMITDATE columns for the existence check in the query. As L_RECEIPTDATE and L_COMMITDATE are compared by this check and no pre-filtering takes place, both columns are fully materialized, meaning that each row of their data vectors is accessed sequentially.

The full run of all queries is dominated by $54.7 \%$ scanned data vector entries and $6.1 \%$ dense data vector accesses. Only $2.8 \%$ of all accesses are random point accesses to the data vectors. $35.1 \%$ of all

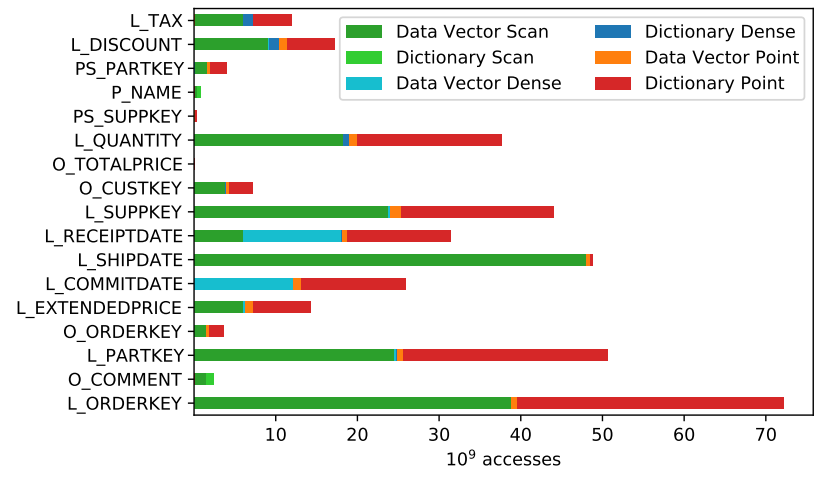

Figure 4: Accesses on the largest used columns for a run of all TPC-H queries.

accesses are dictionary point accesses, and just $1.2 \%$ are dictionary scans or dense dictionary point accesses. Given that we expect scans to be affected less by placing data in NVM, this matches the results in Figure 2 where we observed major slowdowns mostly when placing dictionaries in NVM.

\subsection{Placement Based on Access Counters}

Next, we derive a placement strategy based on the access counters presented in Section 3.3. Ideally, this strategy would follow a quantitative approach based on a cost model that estimates workload performance for a given placement configuration by taking into account the access counters. Using such a model, it would be possible to trade off lower DRAM usage (cost) with performance by translating the problem to a linear optimization problem similar to the LOPT strategy proposed by Vogel et al. [21] for placing data in a tier-less pool of storage devices. However, creating this cost model would also require carefully micro-benchmarking the access types represented by the access counters, which is out of the scope of this work. Additionally, such an approach could potentially also lead to overfitting and result in low robustness against changes in the workload like running previously unknown queries. For these reasons, we omit this step in this paper, and leave it as future work.

We instead decide for a more simple, qualitative approach and propose a heuristic to place the data vector and dictionary of each column according to the accesses to them, with the goal of placing as much data as possible in NVM while deteriorating performance as little as possible. For the heuristic, we consider two main factors: First, we observed in Section 3.3 that more than $70 \%$ of all accesses in a full run of all TPC-H queries are scan-like, and we also identified that this access pattern exhibits similar performance on DRAM and NVM, while a random point access pattern results in a performance loss when data is placed in NVM. Thus, one factor in the heuristic should be the scan-to-point-access-ratio of a data structure. Second, we find that many of the columns with the highest memory consumption are rarely accessed. This is illustrated in Figure 4, where we show the access counters in a run of all TPC-H queries on the largest used columns which constitute more than $90 \%$ of the memory usage of all columns. Examples of infrequently accessed columns are 0_TOTALPRICE, PS_SUPPKEY, 
and P_NAME. As these columns have a large memory usage but are accessed infrequently, it is sensible to design the heuristic such that the data vectors and dictionaries of these columns are placed in NVM regardless of their scan-to-point-access ratio.

Given the number of scanned entries $n_{\text {scan }}$, the number of dense point accesses $n_{\text {dense }}$, and the number of random point accesses $n_{\text {point }}$ for any data vector or dictionary, we propose the following heuristic to decide if the structure should be placed in NVM:

$$
\begin{aligned}
\text { Place in NVM }= & \left(n_{\text {scan }}+n_{\text {dense }}\right)>10 \cdot n_{\text {point }} \| \\
& \left(n_{\text {scan }}+n_{\text {dense }}+n_{\text {point }}\right)<0.01 \cdot n_{\text {total }}
\end{aligned}
$$

where $n_{\text {total }}$ is the total number of accesses on this type of data structure across all columns. Informally, if a structure is scanned ten times more than it is access by random point accesses, or if it is overall accessed by less than $1 \%$ of all accesses to this structure type across the entire workload, we place it in NVM. Note that the factors 10 and $1 \%$ here are somewhat arbitrary and derived experimentally. As noted above, we would ideally rely on micro-benchmarks to factor in the relative performance differences of scans and point accesses, but we skipped this step due to a lack of time. In the future, this could be a good way to improve the heuristic and to enable specific, user-driven cost-performance trade-offs.

\section{EVALUATION}

We now evaluate the proposed placement heuristic by measuring the overall effectiveness of the heuristic and the robustness of the resulting placement configurations against workload changes.

\subsection{Experimental Setup}

Hardware Configuration. We evaluate on a system with two Intel $^{\circledR}$ Xeon $^{\circledR}$ Platinum 8260L CPUs @ 2.40GHz, running SLES 12 SP4. Execution is limited to a single socket to avoid NUMA effects. Per socket, the system has 96 GB of DDR4-2666 DRAM (six 16 GB DIMMs) and 1.5 TB of Intel ${ }^{\circledR}$ Optane $^{\mathrm{TM}} 100$ Series Persistent Memory (six 256 GB DIMMs). Each NVM DIMM shares a CPU memory channel with one DRAM DIMM, which means that using only DRAM reaches the same bandwidth that would be reached with only DRAM in the system. The NVM DIMMs are configured in App Direct (uncached) mode, where reads and writes go directly from the CPU to the NVM DIMM.

Workload. We evaluate the proposed placement heuristic using the TPC-H benchmark at scale factor 100. Like in previous work [21], we define one run as the sum of the measurements of the runtimes of each query executed once sequentially. To obtain stable results we always measure ten consecutive runs and report the mean. The same applies for measurements of individual queries. For the TPC-H dataset, we use SAP HANA's default compression settings and indices on the primary keys of all tables. For certain compressed columns, HANA also automatically creates block indices [13], which could not be made page-loadable at the time of writing. Therefore these indices are never placed in NVM in our experiments, and their memory usage is not included in the totals reported below. Block indices have a DRAM consumption of $12.4 \%$ in addition to the totals reported in Figure 5. The regular primary-key indices are page-loadable and hence stored in DRAM or NVM (in the NVM only configuration) and their memory usage is counted towards the reported values.

We consider this workload choice sensible, as we focus purely on read-optimized main data in this paper. A transactional workload that stresses the write-optimized delta is not of interest here as we did not make any changes to this part of the system.

Software Configuration. As discussed in Section 3.1, we implemented the placement of data structures in NVM by extending SAP HANA's buffer cache, using the built-in NVM block manager (see [1]). All experiments are conducted using this prototype which is otherwise fully equivalent to the SAP HANA Cloud Edition from Q4 2020. We sized the buffer cache sufficiently large such that no data is evicted and that all data used by queries is preloaded into the cache before running queries. All queries are executed using the HANA Execution Engine (HEX), SAP HANA's state-of-the-art code-generating query execution engine.

When all data is placed in NVM, we observe that more instructions than in the DRAM-only configuration are executed during query processing. These additionally executed instructions can be attributed to dynamic optimizations internal to HEX, which lead to a slightly different query execution when data is placed in NVM. For a fair comparison between NVM and DRAM, we take measures to reduce this effect as far as possible without negatively affecting runtime on DRAM, but still arrive at $7 \%$ additionally executed instructions when all data is placed in NVM. This also applies to a lesser extent for the configurations where data is partly placed in NVM, and may lead to an inflation of the measured negative performance impact of NVM. Nevertheless, the NVM-DRAM comparison in this evaluation remains representative, as the performance impacts of placing data in NVM are far beyond what could be caused by the additionally executed instructions.

\subsection{Experiments}

The following experiments evaluate the proposed placement heuristic on queries from the TPC-H benchmark.

Counter-Based Placement. Figure 5a shows the results of applying the proposed counter-based placement heuristic $(\star)$ to a run of all TPC-H queries alongside the previously examined simple strategies. In the experiment, we first performed a training run to collect the access counters, which were then used to place all data vectors and dictionaries using the heuristic from Section 3.4. The results labeled counter-based $(\star)$ were then obtained by performing another run with this placement configuration. In the full run of all queries, the heuristic placed $76 \%$ of the used data in NVM which incurs a slowdown of overall workload performance of less than $5 \%$ relative to execution with all data in DRAM (O). This is an improvement over the simple strategy of placing all data vectors in NVM $(+)$, and is achieved by placing infrequently accessed dictionaries in NVM, in addition to most of the data vectors. The heuristic places all data vectors except those of L_DISCOUNT, L_EXTENDEDPRICE, and O_CUSTKEY in NVM. Not placing these data vectors in NVM results in a slight performance improvement over the strategy that places all data vectors in NVM $(+)$. By additionally placing infrequently accessed dictionaries in NVM, DRAM usage is reduced further than by the simple strategy. 

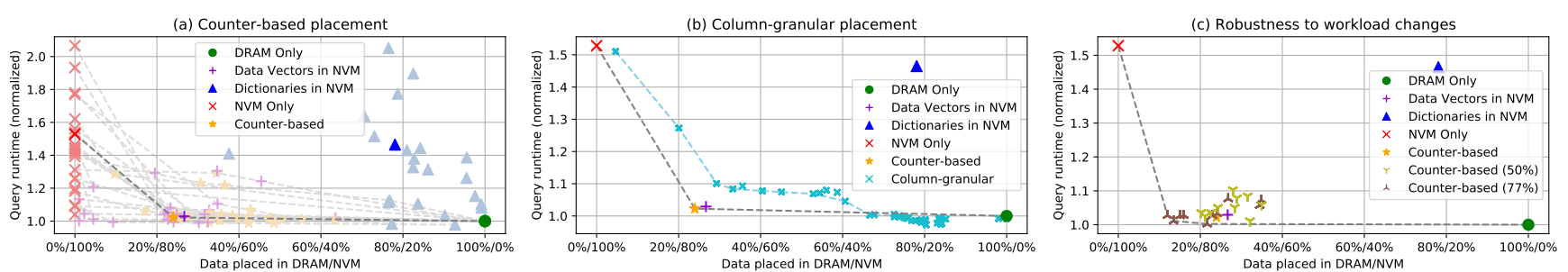

Figure 5: Results for TPC-H SF 100.
Considering the individual queries shown in Figure 5a as discolored entries, it can be seen that, like with the data vector strategy $(+)$, the counter-based strategy $(\star)$ results in more than $20 \%$ slowdown for some queries. In cases where a slowdown is unacceptable for individual queries, this could be addressed by evaluating the placement heuristics on the access counters for just that query, and using its DRAM placement decisions to override NVM placement decided by the heuristic on the access counters for the entire workload. For the overall workload, this could result in a less optimal result. However, we do not explore this direction further, as we are only interested in an optimal strategy for the total workload.

To demonstrate the benefit of placing data at the granularity of data vectors and dictionaries, we compare the results for the full run of all queries with ones obtained using column-granular placement $(\times)$ in Figure 5b. For this, we successively place the columns with the lowest numbers of overall point accesses in NVM, until all columns accessed by the queries are placed in NVM. Note: This does not fully reach the NVM-only configuration $(x)$, as indices are not included in the column-granular placement. The results clearly show that fine-grained placement is superior to column-granular placement. When the same amount of data is placed in NVM as is suggested by the heuristic $(\star)$ with column-granular placement $(\times)$, runtime increases by more than $20 \%$.

Robustness to Workload Changes. To evaluate the robustness of the placement heuristic, we emulate the presence of unknown queries in the workload. For this, we randomly select a subset of the 22 TPC-H queries as calibration queries. Based on their access counters, we then find a placement configuration that is used to evaluate a full run of the 22 queries. Data structures that are not accessed by any of the calibration queries are placed in DRAM, as their suitability for placement in NVM cannot be determined. We found that placing these not-accessed structures in NVM would generally lead to significantly deteriorated performance. Given the general trends observed in accesses on data vectors and dictionaries, it could also be sensible to place only not-accessed data vectors in NVM, but we have not tested this. We run this experiment for ten random sets of 11 and 17 calibration queries. This gives us an understanding of the robustness of our heuristic when only $50 \%$, or respectively $77 \%$ of all queries are known at the time of the placement decision.

Figure $5 \mathrm{c}$ shows the results with the 20 new configurations in addition to the previously discussed results. We observe that even if the heuristic is fed the access counters of $50 \%$ of all queries $(Y)$, the results for all queries still remain in a usable range. More than
$60 \%$ of the data is placed in NVM, and overall performance does not degrade by more than $20 \%$ compared to the DRAM-only (O) configuration. The results also seem to get better when more queries are known to the heuristic. When $77 \%$ of the queries are known ( $\curlywedge$ ), the obtained placement configurations generally place more data in NVM and exhibit better performance than the ones with only $50 \%$ of all queries known.

Several configurations also outperform the configuration determined by the heuristic with full knowledge of all queries $(\star)$. This demonstrates that there is further potential to push the Paretofrontier with data-structure-grained placement. To this end, we discuss directions which we hope to explore further in Section 4.3.

\subsection{Outlook and Discussion}

We demonstrated the effectiveness of a counter-based heuristic for the placement of columnar main data, and the benefits of placing data at the granularity of the column-store data structures, instead of at column-granularity. Despite the already promising results, several open questions and future work remain:

First, we mentioned the possibility of deriving a proper cost model for the counter-based placement decision based on microbenchmarks in Section 3.4. We plan to explore this direction to find out if this can yield better results for the current goal (placing as much data in NVM with as little performance degradation as possible), and to also enable targeted, user-driven trade-offs between cost and performance. In this context more fine-granular placement of horizontally partitioned data could potentially also lead to further improved results. Second, the evaluation of this paper is based solely on the TPC-H benchmark. Evaluating other workloads would be desirable, e.g., mixed HTAP workloads with significant numbers of point lookups, to determine the suitability of NVM for main data in such cases, and to evaluate the effectiveness of the proposed heuristic in particular. As seen in Figure $5 c$, the proposed heuristic does not necessarily produce the most optimal result, and we cannot judge its usefulness for other workloads at this point. For example, the flat threshold of $1 \%$ of all accesses under which a structure is placed in NVM may be too high for workloads where more columns exist, as this would reduce the average access probability for each individual column. Creating and utilizing a more comprehensive cost model to guide placement decisions may yield better results, and evaluating this fine-granular placement on other workloads than TPC-H will likely also spark further insights.

Third, in this paper, we have focused entirely on the placement of data without considering other system parameters or components which could be adjusted to produce more desirable results. 
Interesting aspects in this direction would be the interaction between data compression and data placement, or taking into account where data is located during query optimization. Lastly, a more general question is how or if data placement alternatives like the ones presented here should be exposed to users. While one option would be to offer data placement as a tuning knob or providing a placement advisor, a "self-driving" [18] approach where these decisions are made autonomously by the system without user intervention may be more desirable. In our current implementation, the process of collecting the access counters and using them with the heuristic to generate a placement decision happens outside the DBMS. Integrating this as a closed-loop online solution is desirable, and could also allow for adjusting to workload changes by moving data between memory hierarchy levels as the workload changes.

Finally, while we have implemented and evaluated our ideas in SAP HANA for this paper, our findings should be applicable to other systems as well, especially to other column stores that employ domain encoding. Even in other systems however, it could be possible to find NVM-friendly access patterns at smaller granularities than that of data structures considered here, for example for chunks of rows. Then similarly to what was presented here, such substructures could be placed in NVM without incurring performance degradations.

\section{CONCLUSION}

In this paper we explored the placement of column-store main data structures in NVM, with the goal of placing as much data in NVM as possible while impacting query runtime as little as possible. For this, we observed significantly varying effects on performance when placing different types of data structures in NVM. We showed that this can be attributed to the underlying memory access patterns on these data structures, namely scan and point accesses. Based on this finding we proposed a heuristic that leverages access counters which differentiate between both fundamental access types to guide the placement of data at the granularity of individual column data structures. Our evaluation with the TPC-H benchmark showed that data placement using this heuristic is able to place $76 \%$ of the used data in NVM with a performance impact of less than 5\% compared to a configuration where all data is located in DRAM. In contrast, placing all data in NVM would result in a 53\% slowdown. We also discussed possible directions for future work which we hope to explore further.

\section{ACKNOWLEDGMENTS}

We would like to thank Intel for providing the test system.

\section{REFERENCES}

[1] Mihnea Andrei, Christian Lemke, Günter Radestock, Robert Schulze, Carsten Thiel, Rolando Blanco, Akanksha Meghlan, Muhammad Sharique, Sebastian Seifert, Surendra Vishnoi, Daniel Booss, Thomas Peh, Ivan Schreter, Werner Thesing, Mehul Wagle, and Thomas Willhalm. 2017. SAP HANA Adoption of Non-Volatile Memory. Proc. VLDB Endow. 10, 12 (Aug. 2017), 1754-1765. https://doi.org/10.14778/3137765.3137780

[2] Mihnea Andrei, Christian Lemke, Günter Radestock, Robert Schulze, Carsten Thiel, Rolando Blanco, Akanksha Meghlan, Muhammad Sharique, Sebastian Seifert, Surendra Vishnoi, Daniel Booss, Thomas Peh, Ivan Schreter, Werner Thesing, Mehul Wagle, and Thomas Willhalm. 2017. SAP HANA Adoption of Non-Volatile Memory. Proc. VLDB Endow. 10, 12 (Aug. 2017), 1754-1765. https://doi.org/10.14778/3137765.3137780
[3] Joy Arulraj, Justin Levandoski, Umar Farooq Minhas, and Per-Ake Larson. 2018. BzTree: A High-Performance Latch-Free Range Index for Non-Volatile Memory. Proc. VLDB Endow. 11, 5 (Jan. 2018), 553-565. https://doi.org/10.1145/3164135. 3164147

[4] Joy Arulraj and Andrew Pavlo. 2017. How to Build a Non-Volatile Memory Database Management System. In Proceedings of the 2017 ACM International Conference on Management of Data (Chicago, Illinois, USA) (SIGMOD '17). Association for Computing Machinery, New York, NY, USA, 1753-1758. https://doi.org/10.1145/3035918.3054780

[5] Joy Arulraj, Andy Pavlo, and Krishna Teja Malladi. 2019. Multi-Tier Buffer Management and Storage System Design for Non-Volatile Memory. CoRR abs/1901.10938 (2019). arXiv:1901.10938 http://arxiv.org/abs/1901.10938

[6] Bishwaranjan Bhattacharjee, Mustafa Canim, Christian A Lang, George A Mihaila, and Kenneth A Ross. 2010. Storage Class Memory Aware Data Management. IEEE Data Eng. Bull. 33, 4 (2010), 35-40.

[7] Martin Boissier, Rainer Schlosser, and Matthias Uflacker. 2018. Hybrid Data Layouts for Tiered HTAP Databases with Pareto-Optimal Data Placements. In 2018 IEEE 34th International Conference on Data Engineering (ICDE). IEEE, Piscataway Township, NJ, USA, 209-220. https://doi.org/10.1109/ICDE.2018.00028

[8] Michael Brendle, Nick Weber, Mahammad Valiyev, Norman May, Robert Schulze, Alexander Böhm, Guido Moerkotte, and Michael Grossniklaus. 2021. Precise, Compact, and Fast Data Access Counters for Automated Physical Database Design. BTW 2021 (2021).

[9] Shimin Chen and Qin Jin. 2015. Persistent $B^{+}$-Trees in Non-Volatile Main Memory. Proc. VLDB Endow. 8, 7 (Feb. 2015), 786-797. https://doi.org/10.14778/2752939. 2752947

[10] Justin DeBrabant, Joy Arulraj, Andrew Pavlo, Michael Stonebraker, Stan Zdonik, and Subramanya Dulloor. 2014. A prolegomenon on OLTP database systems for non-volatile memory. ADMS@VLDB (2014).

[11] Franz Färber, Norman May, Wolfgang Lehner, Philipp Große, Ingo Müller, Hannes Rauhe, and Jonathan Dees. 2012. The SAP HANA Database-An Architecture Overview. IEEE Data Eng. Bull. 35, 1 (2012), 28-33.

[12] Joseph Izraelevitz, Jian Yang, Lu Zhang, Juno Kim, Xiao Liu, Amirsaman Memaripour, Yun Joon Soh, Zixuan Wang, Yi Xu, Subramanya R. Dulloor, Jishen Zhao, and Steven Swanson. 2019. Basic Performance Measurements of the Intel Optane DC Persistent Memory Module. CoRR abs/1903.05714 (2019). arXiv:1903.05714 http://arxiv.org/abs/1903.05714

[13] Christian Lemke, Kai-Uwe Sattler, Franz Faerber, and Alexander Zeier. 2010. Speeding Up Queries in Column Stores. In Data Warehousing and Knowledge Discovery. Springer Berlin Heidelberg, Berlin, Heidelberg, 117-129.

[14] Lucas Lersch, Wolfgang Lehner, and Ismail Oukid. 2019. Persistent Buffer Management with Optimistic Consistency. In Proceedings of the 15th International Workshop on Data Management on New Hardware (Amsterdam, Netherlands) (DaMoN'19). Association for Computing Machinery, New York, NY, USA, Article 14, 3 pages. https://doi.org/10.1145/3329785.3329931

[15] Ingo Müller, Cornelius Ratsch, Franz Faerber, et al. 2014. Adaptive String Dictionary Compression in In-Memory Column-Store Database Systems.. In EDBT, Vol. 14. 283-294.

[16] Ismail Oukid, Daniel Booss, Wolfgang Lehner, Peter Bumbulis, and Thomas Willhalm. 2014. SOFORT: A Hybrid SCM-DRAM Storage Engine for Fast Data Recovery. In Proceedings of the Tenth International Workshop on Data Management on New Hardware (Snowbird, Utah) (DaMoN '14). Association for Computing Machinery, New York, NY, USA, Article 8, 7 pages. https://doi.org/10.1145/ 2619228.2619236

[17] Ismail Oukid, Johan Lasperas, Anisoara Nica, Thomas Willhalm, and Wolfgang Lehner. 2016. FPTree: A Hybrid SCM-DRAM Persistent and Concurrent B-Tree for Storage Class Memory (SIGMOD '16). Association for Computing Machinery, New York, NY, USA, 371-386. https://doi.org/10.1145/2882903.2915251

[18] Andrew Pavlo, Gustavo Angulo, Joy Arulraj, Haibin Lin, Jiexi Lin, Lin Ma, Prashanth Menon, Todd C Mowry, Matthew Perron, Ian Quah, et al. 2017. SelfDriving Database Management Systems.. In CIDR, Vol. 4. 1.

[19] Reza Sherkat, Colin Florendo, Mihnea Andrei, Rolando Blanco, Adrian Dragusanu, Amit Pathak, Pushkar Khadilkar, Neeraj Kulkarni, Christian Lemke, Sebastian Seifert, Sarika Iyer, Sasikanth Gottapu, Robert Schulze, Chaitanya Gottipati, Nirvik Basak, Yanhong Wang, Vivek Kandiyanallur, Santosh Pendap, Dheren Gala, Rajesh Almeida, and Prasanta Ghosh. 2019. Native Store Extension for SAP HANA. Proc. VLDB Endow. 12, 12 (Aug. 2019), 2047-2058. https://doi.org/10. 14778/3352063.3352123

[20] Alexander van Renen, Viktor Leis, Alfons Kemper, Thomas Neumann, Takushi Hashida, Kazuichi Oe, Yoshiyasu Doi, Lilian Harada, and Mitsuru Sato. 2018. Managing Non-Volatile Memory in Database Systems. In Proceedings of the 2018 International Conference on Management of Data (Houston, TX, USA) (SIGMOD '18). Association for Computing Machinery, New York, NY, USA, 1541-1555. https://doi.org/10.1145/3183713.3196897

[21] Lukas Vogel, Viktor Leis, Alexander van Renen, Thomas Neumann, Satoshi Imamura, and Alfons Kemper. 2020. Mosaic: A Budget-Conscious Storage Engine for Relational Database Systems. Proc. VLDB Endow. 13, 12 (July 2020), 2662-2675. https://doi.org/10.14778/3407790.3407852 
[22] Thomas Willhalm, Nicolae Popovici, Yazan Boshmaf, Hasso Plattner, Alexander Zeier, and Jan Schaffner. 2009. SIMD-Scan: Ultra Fast in-Memory Table Scan Using on-Chip Vector Processing Units. Proc. VLDB Endow. 2, 1 (Aug. 2009), 385-394. https://doi.org/10.14778/1687627.1687671
[23] Krishna Yalamanchi. 2021. Turbocharge your S/4HANA experience with Optane Persistent Memory. https://community.intel.com/t5/Intel-OptanePersistent-Memory/Turbocharge-your-S-4HANA-experience-with-OptanePersistent/ba-p/1263628 Last accessed: 2021-03-25. 